\title{
Laparoscopic lymph nodes dissection for advanced gastric cancer: the current status and the perspective
}

\author{
Masanari Shimada, Susumu Amaya, Yoshinori Munemoto, Takeshi Mitsui \\ Department of Surgery, Fukui-ken Saiseikai Hospital, Fukui 918-8503, Japan. \\ Correspondence to: Dr. Masanari Shimada, Department of Surgery, Fukui-ken Saiseikai Hospital, Wadanaka Funabashi 7-1, \\ Fukui 918-8503, Japan. E-mail: masanari.shimada@gmail.com \\ How to cite this article: Shimada M, Amaya S, Munemoto Y, Mitsui T. Laparoscopic lymph nodes dissection for advanced gastric \\ cancer: the current status and the perspective. Mini-invasive Surg 2019;3:7. http://dx.doi.org/10.20517/2574-1225.2018.78
}

Received: 19 Dec 2018 First Decision: 18 Feb 2019 Revised: 25 Feb 2019 Accepted: 26 Feb 2019 Published: 27 Mar 2019

Science Editor: Tetsu Fukunaga Copy Editor: Cai-Hong Wang Production Editor: Huan-Liang Wu

\begin{abstract}
The laparoscopic gastrectomy (LG) with D2 lymph node dissection (LND) for advanced gastric cancer (AGC) have been widely done. However, the applicability to more advanced disease is still under debate. Actually, there are a lot of technical demands against D2 LND for AGC, e.g., total omentectomy, splenic hilar node dissection, and the management for bulky lymph nodes, etc. Recently, extensive research has been gradually performed in the field of LG for AGC and demonstrated that LG for AGC is a safe and feasible procedure with better short-term outcomes compared with open gastrectomy. Also, large-scaled phase III trials are ongoing, and their long-term outcomes are awaited the publication in the near future. LG with D2 LND by expert surgeons under the cautious indications could be acceptable treatment for locally AGC. On the other hand, we should keep searching for solutions to the technical or oncological issues, and long-term outcome of phase III study should be warranted for standard treatment. Robotic surgery, LG following neoadjuvant chemotherapy, or conversion therapy using LG for several stage IV patients may help us clear the technical hurdles, and may show survival advantages in the future.
\end{abstract}

Keywords: Laparoscopic gastrectomy, advanced gastric cancer, lymph node dissection, distal gastrectomy, total gastrectomy

\section{INTRODUCTION}

Laparoscopic gastrectomy (LG) for gastric cancer has been popular rapidly with the improvement of technique and the progress of surgical devices. In the latest Gastric Cancer Treatment Guidelines 2018 (ver.5) published by Japanese Gastric Cancer Association ${ }^{[1]}$, laparoscopic distal gastrectomy (LDG) for clinical

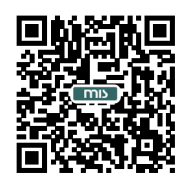


stage I disease is accepted for one of the options in daily clinical practice. Recently, extensive research has been gradually performed in the field of LG for advanced gastric cancer (AGC) and demonstrated that LG for AGC is a safe and feasible procedure with better short-term outcomes compared with open gastrectomy (OG). However, there are few randomized clinical trials (RCT) reporting the long-term outcome of LG for AGC previously. Moreover, it also remains controversial whether LG can be performed for AGC from the aspect of technical and oncological issues. Clinically, we often encounter the situations that the disease is unexpectedly diagnosed with advanced disease in laparoscopic inspection. Moreover, if it comes to that the histological examination may reveals serosa invasions or multiple lymph node metastases even if only D1 or D1+ lymph node dissection (LND) has been done because of the clinical stage I, we are sorry D2 LND had not been performed in such cases. So, surgeons must prepare to perform D2 LND in laparoscopic gastrectomy and recognize the acceptable indications and limitations for AGC. Now, we summarized the main points of surgical procedure of D2 LND and the future perspectives.

\section{PREVIOUS STUDIES ABOUT RADICAL LG FOR AGC}

Recently, many retrospective comparative studies and several prospective RCTs have reported that LG for AGC was safe and feasible when compared to the short-term and long-term outcomes observed with $\mathrm{OG}^{[2-13]}$. Table 1 summarizes these studies about LG for AGC in recent years. Propensity score matching analysis (PSM) was often used for comparison the LG and OG groups in some retrospective studies ${ }^{[8,10,12]}$. Especially, some authors demonstrated the technical safety of LDG with D2 LND for locally AGC in the multi-institutional, prospective, phase II study ${ }^{[2-5,11]}$. Moreover, the 3-year or 5-year overall or disease-free survival rates have been gradually reported from China, Korea, and Japan ${ }^{[7-13]}$. Majority of them reported that LG is feasible and safe for the treatment of AGC with D2 LND compared with OG, and no significant differences were observed in long-term over all survival (OS) and disease free survival (DFS) between the LG group and OG group. However, Li et al. ${ }^{[6]}$ suggested higher-level tumor stage may increase the operative risk and should be performed with caution by surgeons with considerable experience of LG. Also, Lin $e t a l{ }^{[8]}$ reported although the OS curve at each stage did not differ significantly, the survival rate increased overall for patients with T4aN3bMo in the OG group. Additionally, because the patient selections in their studies are different slightly in each and there is the difference that laparoscopic total gastrectomy (LTG) is embedded in the studies or not, we should give the result careful consideration. Collectively, it seems that LG with D2 LND could be acceptable treatment for AGC under definite conditions by expert surgeons.

\section{CLINICAL INDICATIONS AND LIMITATIONS DEALING WITH LND FOR AGC}

\section{Tumor infiltration}

Recently, the result of JCOG1001 (UMIN000003688) has been published, which demonstrated no survival difference between omentectomy $v s$. bursectomy for T3/T4 tumors diagnoses with surgical findings in $\mathrm{OG}^{[14]}$. Therefore, bursectomy is not recommended as a standard procedure for AGC in Japan. However, the significance for omentectomy only does not become clear yet, because it is determined that omentum should be resected in both omentectomy group and bursectomy group in JCOG1001 study. Some reports indicated that in some metastatic nodes extra-nodal expansion is recognize, which means cancer cell spread out of lymph node capsule to adjacent adipose tissue ${ }^{[15,16]}$. Extra-nodal expansion is pointed out to be a poor prognostic factor ${ }^{[15]}$. Based on these reports, if the prognosis will be improved with omentectomy, it is expected to be significant clinically for T4 tumor. Then, omentectomy is performed for patients having tumors deeper than T3 in a lot of institutes at the moment. Presently, RCT is scheduled to launch, which validate the non-inferiority of omentum-preserving surgery for $\mathrm{T} 3 / \mathrm{T} 4$ tumors.

\section{Bulky positive nodes and large primary tumor}

Generally, bulky lymph node is, by definition, "one node $\geq 3 \mathrm{~cm}$ in diameter" or "nearby more than one nodes $\geq 1.5 \mathrm{~cm}$ in diameter", and neoadjuvant chemotherapy (NAC) is often performed in such patients. 


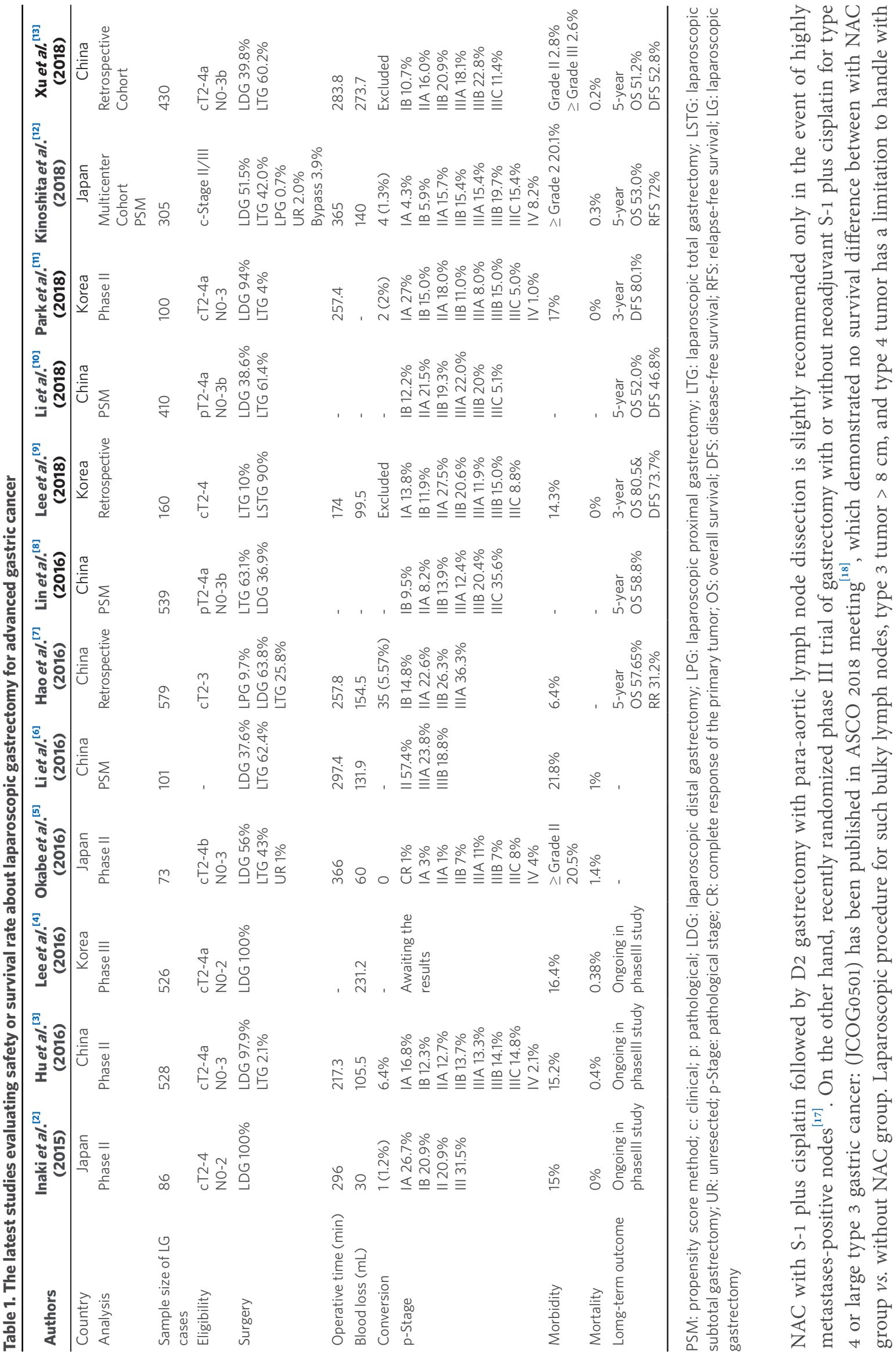


endoscopic forceps with concerns of spillage of cancer cells. Thus, primary open surgery with D2 LND and adjuvant chemotherapy remains the standard treatment for type 4 or large type 3 tumors.

Probably, majority of surgeons will be convinced of the indication of LG about positive nodes and large tumor as below: (1) lymph nodes are not bulky; (2) no invasion to other organ or major vessels; (3) tumor size is less than $8 \mathrm{~cm}$ in diameter; and (4) non-type 4 tumor. In that context, Okabe $e t$ al. ${ }^{[5]}$ disclosed a phase II study (KUGC04), which demonstrated safety and efficacy of LG for gastric cancer of clinical stage II or higher, including patients with prior chemotherapy, tumors requiring TG, tumors that invaded adjacent organs, and patients with bulky nodes metastasis. Solid evidence of the surgical and oncological safety of LG for AGC requires performance of a multicenter, prospective study with experienced surgeons.

\section{Splenic hilar dissection for proximal gastric cancer}

In Japan, splenic hilar nodes (No.10) have been included within the extent of D2 LND in the treatment of proximal AGC for a long time. However, the final result of JCOG 0110 (UMINC000000004) has been disclosed, which compared splenectomy vs. non-splenectomy for proximal AGC not invading greater curvature line. There was no difference in long-term survival rate. Furthermore, splenectomy was associated with increased incidence of morbidity ${ }^{[19]}$. Therefore, splenectomy is not recommended as a standard treatment, except for tumors invading the greater curvature line. Conversely, there is a possibility that such tumors invading the greater curvature line or tumors with metastases of splenic hilar lymph node may be indicated for splenic hilar dissection. Laparoscopic approach has a great advantage for procedures in deep surgical fields around spleen. However, for complicated cases, such as invading the splenogastric ligament or the pancreatic tail, there are strong doubts about whether laparoscopic maneuver is applicable or not.

\section{THE TECHNICAL TIPS OF LND FOR AGC}

\section{Preoperative evaluation}

Preoperative esophagogastroduodenoscopy, contrast enhanced computed tomography, and positron emission tomography are important for accurate diagnosis on tumor depth, invasion adjacent organs, lymph node metastases, or distant metastases. Three-dimensional computed tomography is also helpful to recognize the branching of celiac artery or anatomical diversity of the splenic hilar vessels ${ }^{[20]}$.

\section{Positions of trocars}

Reverse Trendelenberg position with head elevated about $15-20^{\circ}$. The surgeon stands on the patient's right side, the assistant is on the left side, and the assistant for camera stand between the patient's legs. A scope port is inserted via umbilical mini-laparotomy. For manipulation, $5 \mathrm{~mm}$ trocars are inserted on bilateral subcostal midclavicular line, and $12 \mathrm{~mm}$ trocars are inserted on bilateral lateroabdominal region, which arranged in an inverted trapezoidally. Especially, because the raised pancreatic head or vertebral body get in the way of dissection in case of dissection around esophagus or deep suprapancreatic lymph node dissection, right lateroabdominal trocar should be arranged slightly medially and cranially.

\section{Laparoscopic inspection}

At first, it is identified that there is no metastasis on the surface of the liver and peritoneal dissemination in the omentum, mesocolon, and mesenterium. Subsequently, intraoperative cytology of ascites in the pelvic cavity or peritoneal lavage specimen is examined. If the intraoperative cytology detects free cancer cells, it is considered to be "non-curative factor (CY1)". However, if there is no "non-curative factor" other than CY1, it is often recommend now that we should convert LG into OG with D2 LND for standard radical surgery and extensive intraoperative peritoneal lavage using a large amount of saline solution.

\section{Dividing the greater omentum and dissection of the gastrosplenic ligament}

Omentectomy is performed for almost patients having tumors deeper than T3. Discussion of the greater omentum is started near transverse colon. Surgeon's left forceps and the assistant's right forceps elevate 


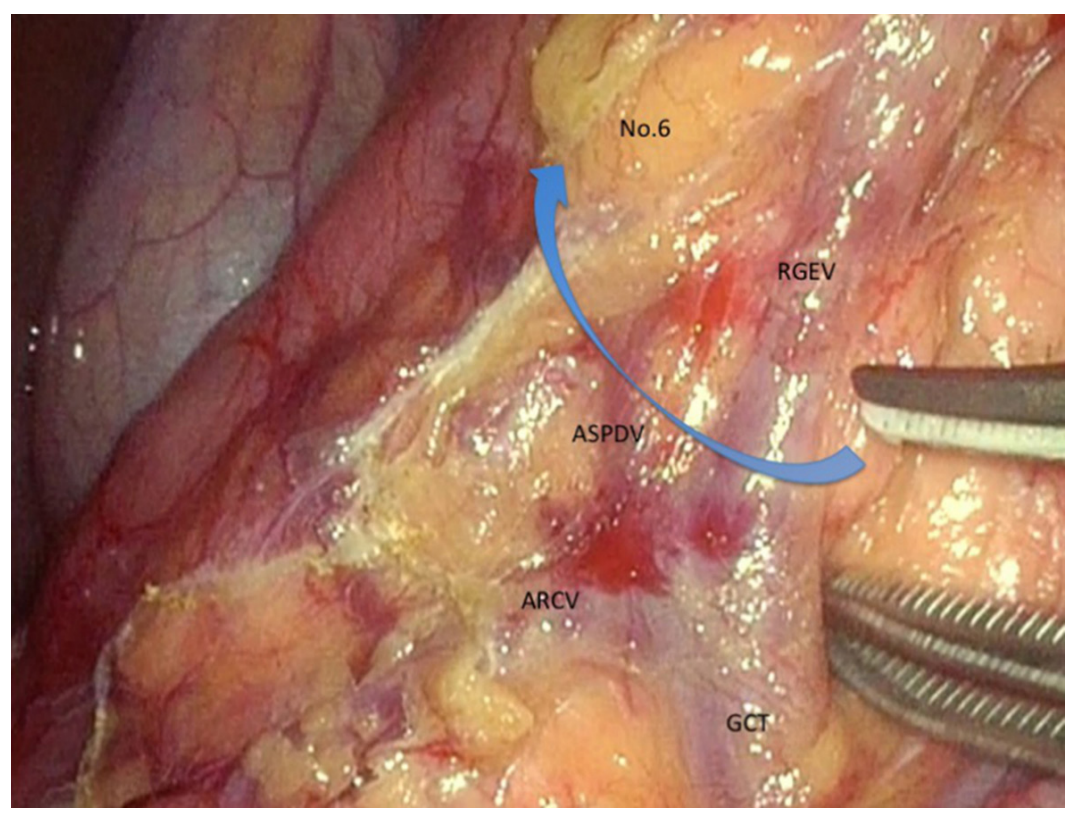

Figure 1. We present the boundary of No.6 lymph node station. The operator should identify anterior superior pancreaticoduodenal vein (ASPDV) and elevates No.6 lymphoid tissues ventrally and softly. ARCV: accessory right colic vein; GCT: gastrocolic trunk; RGEV: right gastroepiploic vein

the greater omentum; moreover, the wide triangulation is formed by the traction of fatty appendices of transverse colon with the assistant's left forceps. The triangulated counter traction makes it possible for surgeons to resect the greater omentum. Then, the splenocolic ligament is divided and the root of left gastroepiploic artery and vein (LGEAV) are identified in the pancreatic tail. After LGEAV are ligated and cut, the gastrosplenic ligament including short gastric artery (SGA) and vein are dissected toward cranial side. Surgeon is careful not to injure the branch around the spleen. In the area of superior border of spleen, because the operative field often becomes limited by excess fatty tissue, in such occasions the precursor cutting of esophagus may be able to show good operative field toward caudal side later. Alternatively the approach that goes into the left gastrophrenic ligament can confirm the superior border of spleen and the most cranial branch of SGA. Therefore, the mobility of the gastrosplenic ligament is improved by cutting the most cranial branch of SGA, and the gastrosplenic ligament is spread like a "screen". This approach is flexible enough to respond to variety of pancreatic tail or splenic hilum ${ }^{[21]}$.

\section{Dissection of infra-pyloric nodes (No.6)}

The surgeon moves to the patient's left side during the dissection of No.6 lymph node station. After the omentectomy has been finished close to the hepatic flexure, the layer of "embryologic failure of fusion" which consists of the anterior lobe of transverse mesocolon and the anterior pancreatic fascia is dissected for the mobilization of transverse colon. Thereby, we are able to finally determine the range to dissect and ready to dissect No.6 lymph node station. The inferior limit of infra-pyloric nodes is anterior superior pancreaticoduodenal vein (ASPDV) [Figure 1]. The right gastroepiploic vein is clipped and cut at the point in which ASPDV flows. Then, the lymph nodes and fat tissue in front of ASPDV is elevated and dissected toward descending duodenum. Subsequently, the neural layer around the right gastroepiploic artery (RGEA), which is called "outermost layer", is identified and dissected keeping the layer. Then, the lymphoid tissue on the left side of RGEA is softly elevated ventrally. Nerve bundle around RGEA is cut and RGEA is clipped and cut. Because the lymphoid tissue around infra-pyloric artery (IPA) remain formed into a screen, IPA is cut and lymphoid tissue attaching to duodenum is peeled up.

\section{Cutting the duodenum}

The assistant lift up the posterior wall of the stomach, and the branches of superior duodenal artery is cut from dorsal side. Then, the duodenal bulb is stapled and cut. In patients suspected of duodenal invasion of 


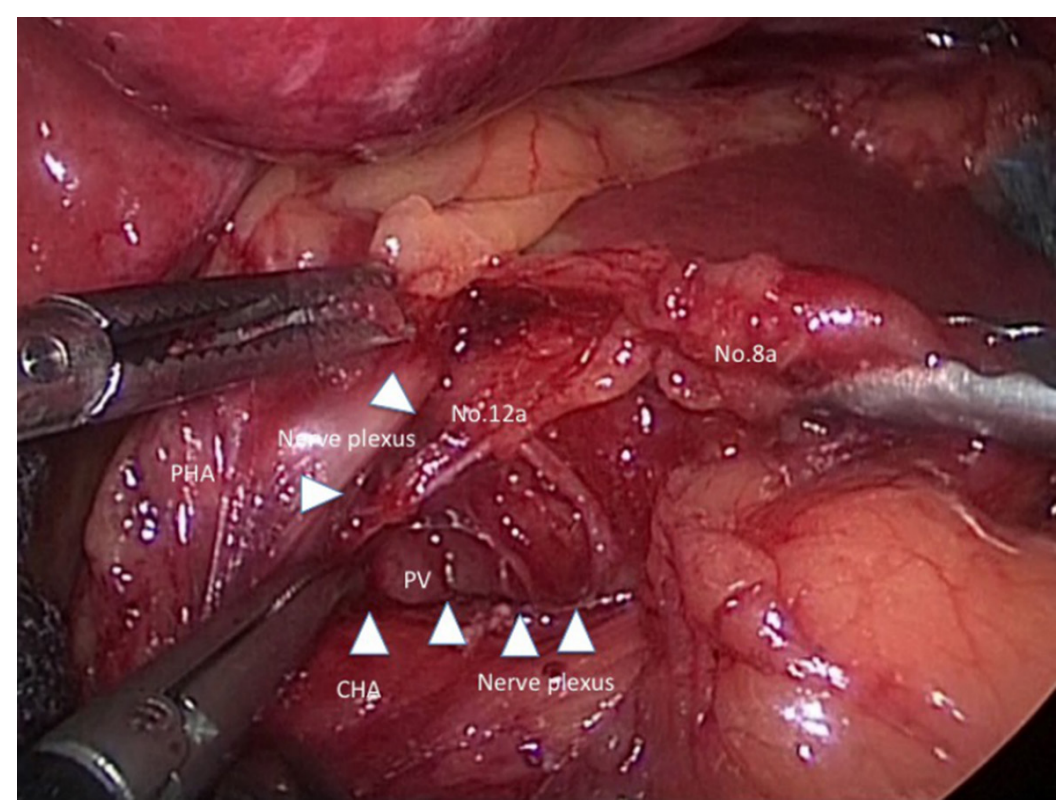

Figure 2. We present a scene in suprapancreatic lymph node dissection. The outermost layer existing between nerve plexus around common hepatic artery (CHA) and No.8a lymph nodes is traced by white arrowheads. PV: portal vein; PHA: proper hepatic artery

gastric cancer, we have to cut the duodenum at the level of superior duodenal angulus. Additionally, the staple line of duodenal stump should be reinforced by intracorporeal suture to prevent the duodenal stump fistula. Recently, it is reported that the method using barbed suture can be performed in a short operation time without any technical difficulties ${ }^{[2]}$.

\section{Dissection of suprapancreatic nodes (No.5, 8a, 9, 12a)}

At first, the pancreatic capsule is cut along the lines with superior edge of pancreas from the root of gastroduodenal artery to near the root of posterior gastric artery (PGA). The assistant's right forceps grasps the gastropancreatic fold ventrally and the left forceps rotates the pancreas dorsocaudally. There are some pancreatic rotating techniques with forceps, gauze, sponge, cotton tc $^{\left[{ }^{[23-26]}\right.}$, however surgeons should avoid the pancreatic injury by delicate compression and coordination with surgeon's devices.

Subsequently, the outermost layer existing between nerve plexus around common hepatic artery (CHA) and No.8a lymph nodes is probed with dissecting forceps [Figure 2]. The ventral side of CHA, proper hepatic artery, and the dorsal side of right gastric artery (RGA) is exposed continuously if the outermost layer is kept dissecting. Then, RGA is clipped and cut at the root and No.5 lymph node dissection is finished.

Next, if the assistant's right forceps grasps and tract No.8a ventrally, the assistant's left forceps and the surgeon's left forceps tracts the nerve plexus around CHA caudally, No.12a lymph node at the hepatoduodenal ligament is pulled out and the left wall of portal vein (PV) is exposed dorsalward. Because the visual reference of PV determine the dorsal limit of No.12a lymph node dissection, the cranial edge of No.12a lymph node is sealed and cut by ultrasonically activated device etc. near hepatic portal region.

Then, the assistant's right forceps grasps and lifts up the gastropancreatic fold ventrally and straight again, and the surgeon's left forceps grasps and lift up the capsule of dissected lymph nodes. The surgeon keeps dissecting from No.8a lymph node to proximal region of splenic artery while he sustains the outermost layer. The left gastric vein is clipped and cut along the way and the left outermost layer of left gastric artery (LGA) is identified preferentially. Herein, the approach that goes into the left gastrophrenic ligament is also 


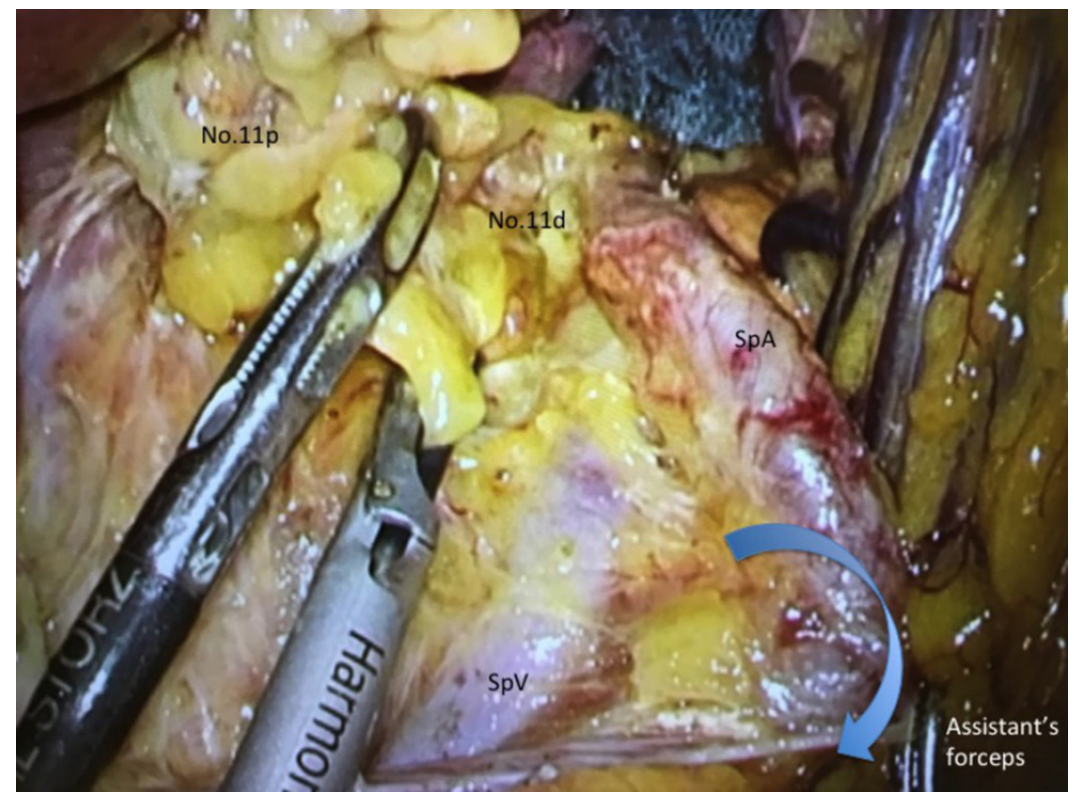

Figure 3. We present a scene in dissection of lymph nodes along splenic artery (SpA) The assistant's left forceps grasps connective tissue around SpA and make SpA straight. Then, we can have visual contact with the dorsal side of the splenic vessels. SpV: splenic vein

effective. In other words, the dissection from the left outermost layer of LGA to crura of the diaphragm is performed at once. The right outermost layer of LGA is similarly identified, and LGA remain celiac nervescovered. Then, LGA and surrounding celiac nerves is double clipped and cut. No.9 lymph node around celiac axis is lead to improvement in mobility by dissecting the outermost layer around the celiac and hepatic nerves, and No.9 lymph node leading to No.16 (para-aortic nodes) should be clipped at the root to prevent lymphorrhea.

Dissection of lymph nodes along splenic artery and splenic hilar nodes (No.11p, 11d, 10)

The most common technical difficulty encountered during LTG with D2 LND is dissection of the lymph nodes among splenic artery (SpA) and splenic hilar lymph nodes (No.10, 11p, and 11d).

At the first setout, the dorsal layer of Toldt's fusion fascia is dissected widely. The assistant's left forceps grasps connective tissue around SpA and make SpA straight; moreover, the assistant's right forceps rotates the lower edge of pancreatic tail dorsally. Then, we can have visual contact with the dorsal side of the splenic vessels [Figure 3], and No.11p and No.11d lymph nodes are dissected toward the root if of LGEAV, which have been initially ligated. Hur et al. ${ }^{[27]}$ reported that taping the splenic artery was helpful in dissecting lymph nodes No.10 and 11d during spleen- and pancreas-preserving LG. The PGA is clipped and cut along the way wherever possible. We should try to preserve the caudal pancreatic artery and vein in the region of pancreatic tail. In splenic hilar region, surgeons are careful not to suffer injuries to pancreatic parenchyma hidden behind the SpA. Finally, the SGA, which arising from the SpA, is clipped and cut at the root.

\section{Throughout the surgery}

The intraoperative characteristics about AGC with metastatic lymph nodes are as below: (1) the Surgical field of view is restricted because of the decline of organ mobility; (2) the identification of the dissectable layer and vessels is difficult; (3) the oozing derives from the fatty tissue around lymph nodes; (4) the mists and fluids, which produced when the energy devices are activated, increase significantly.

In D2 LND for AGC, making an operating field against the metastatic lymph node and the tumor is the first important procedure. We should start dissecting on the normal tissues and keep the dissecting layer 
toward each landmark of surgical scenes, while we try to prevent the organ injury by delicate manipulation. For non-touch isolation of the tumor, gauze is frequently used to retract or lift up stomach, and to absorb bleeding or lymphatic fluid, which can make dry field.

\section{FUTURE PERSPECTIVE}

\section{Ongoing prospective studies}

To provide answers to the extent of laparoscopic LND in AGC, phase III trials to confirm the noninferiority of this procedure to open are ongoing. In Japan, the short-term outcome of the randomized controlled trial to evaluate laparoscopic vs. open surgery for AGC (JLSSG0901) has been published in the 30th annual meeting of the Japan Society for Endoscopic Surgery in December 2017, which demonstrated no significant differences in grade 3 and higher postoperative complications between two groups (3.1\% vs. $4.7 \%{ }^{[28]}$. In China, CLASS-01 (NCT01609309) has been conducted and the short-term outcomes were already published, demonstrating no difference between LG and OG (15.2\%vs. $12.9 \%)$ in morbidity rate ${ }^{[3]}$. The final outcomes, namely 3-year DFS, are awaited the publication before long. In Korea, the short-term outcomes of KLASS-02 (NCT01456598) were disclosed in ASCO 2016 meeting $^{[4]}$, which demonstrated less complication rate ( $16.4 \%$ vs. $24.3 \%$ ), less use of analgesics, and faster recovery in LG group. Its primary endpoint, or 3-year relapse free survival, will be analyzed also anytime soon. We should wait for the final results of these three phase-III trials. Especially, not only long-term survival rate but also difference of recurrence pattern should be carefully checked the specific feature in recurrences after LG. Concerning LTG, a Korean group has launched multicenter randomized controlled trial for application of LTG with LND for gastric cancer (KLASS-06; NCT03385018) in 2018. However, some researchers suggested that LTG for AGC should be carried out on a trial basis until the definitive results are available, and surgeons should be particularly attentive to No.10 or 11d LND without lessening the quality of LND compared with open total gastrectomy ${ }^{[29]}$. The data from these studies are expected to decide future directions for the indication of LG for AGC.

\section{Neo adjuvant chemotherapy}

A few phase III trials and retrospective studies have provided supportive evidence that NAC results in high compliance, as well as other favorable factors such as high rate of Ro resection and tumor regression, which lead to a better prognosis ${ }^{[17,30,31]}$. There have been many RCT comparing LG with OG as mentioned above. However, very few on the comparison between LG + NAC and OG + NAC. Recently, a phase II trial to which evaluate the safety and efficacy of LG after NAC for distal advanced gastric cancer and which provide theoretical basis for conducting a multicenter phase III verification clinical trial conducted in China ${ }^{[32]}$. Long term follow up and piling up the cases will be necessary in the future.

\section{Conversion surgery}

The term "conversion therapy" describes a therapeutic concept in which the treatment strategy is converted by chemotherapy to curative surgery through an oncosurgical approach. The terms "conversion surgery" or "adjuvant surgery" can be applied to the operations performed for conversion therapy. Yoshida et al. ${ }^{[33]}$ proposed that the indications for conversion therapy include patients with marginally resectable metastasis, some patients who are incurable and unresectable except certain circumstances of local palliation needs, and patients with noncurable metastasis in whom an Ro resection can be expected after a satisfactory response to chemotherapy. There were long-term survivors who underwent conversion surgery for such patients. The median survival time of the patients who underwent surgical resection was 30.5 months, as opposed to those who received chemotherapy alone at 11.3 months ${ }^{[34]}$. If the feasibility of this concept will be estimated in the near future by large-scale retrospective and prospective cohort studies, laparoscopic approach may be applied to treatment for minimal invasive surgery.

\section{Robotic surgery}

Robotic surgical instruments seem to have potential to cover disadvantages of LG, such as insufficiency of forceps' degree of freedom or surgeons' physiological tremor at the tip of device ${ }^{[35]}$. It is suggested by 
experts that the use of a surgical robot may be beneficial for more complicated procedures, including more advanced cancer disease ${ }^{[36]}$. Although a number of robot-assisted gastrectomy (RAG) are rapidly increasing since RAG has been covered by insurance in April 2018 in some limited institutes, several issues remain to be solved regarding clinical indication, short- and long-term outcomes, cost- effectiveness, and stress of surgeons ${ }^{[37-39]}$. Recently, Li et al. ${ }^{[40]}$ reported a retrospective PSM analysis that the overall postoperative complication rate was $13.4 \%$ and $11.6 \%$ in the RAG and LG groups, with no significant difference, and the 3-year OS and recurrence rates of the RAG and LG groups were also comparable (78.6\% vs. $74.1 \% ; 18.8 \%$ vs. $21.4 \%$; respectively). Moreover, multicenter prospective study of RAG vs. LG for gastric cancer including AGC has been published in 2016, which demonstrated no significant differences between groups were noted in overall complication and mortality rates, estimated blood loss, rates of open conversion, diet build-up, or length of hospital stay, except for operative time and total costs ${ }^{[41]}$.

Thus, although RAG has evident benefits, it is difficult to assess and compare some advantages at the moment with respect to traditional surgery. Larger randomized prospective trials, well-designed costeffectiveness analysis, and high-quality comparative-effectiveness research are needed before robotic resection can be considered an acceptable alternative for patients with AGC. Probably, the main indication for RAG is when it serves as an adjunct to laparoscopic resection in selected patients with local advanced tumors requiring a D2 LND.

\section{CONCLUSION}

LG with D2 lymph node dissection by expert surgeons under the cautious indications could be acceptable treatment for locally AGC. On the other hand, we should keep searching for solutions to the technical or oncological issues, and long-term outcome of phase III study should be warranted for standard treatment.

\section{DECLARATIONS}

\section{Authors' contributions}

Retrieved the data and cited literatures: Shimada M

Provided technical and clinical advices for Shimada M: Amaya S, Munemoto Y, Mitsui T

Read and approved the final manuscript: All authors

\section{Availability of data and materials}

Not applicable.

\section{Financial support and sponsorship}

None.

\section{Conflicts of interest}

All authors declared that there are no conflicts of interest.

\section{Ethical approval and consent to participate}

Not applicable.

\section{Consent for publication}

Not applicable.

\section{Copyright}

(c) The Author(s) 2019. 


\section{REFERENCES}

1. Japanese Gastric Cancer Association. Gastric Cancer Treatment Guidelines 2018 (ver.5). Tokyo: Kanehara-shuppan; 2018. pp. 17-8. (in Japanese)

2. Inaki N, Etoh T, Ohyama $\mathrm{T}$, Uchiyama $\mathrm{K}$, Katada $\mathrm{N}$, et al. A multi-instistional, prospective, phase II feasibility study of laparoscopyassisted distal gastrectomy with D2 lymph node dissection for locally advanced gastric cancer (JLSSG0901). World J Surg 2015;39:2734-41.

3. Hu Y, Huang C, Sun Y, Su X, Cao H, et al. Morbidity and mortality of laparoscopic versus open D2 distal gastrectomy for advanced gastric cancer: a randomized controlled trial. J Clin Oncol 2016;34:1350-7.

4. Lee HJ, Hyung WJ, Yang HK, Han SU, Park YK, et al. Morbidity of laparoscopic distal gastrectomy with D2 lymphadenectomy compared with open distal gastrectomy for locally advanced gastric cancer: Short term outcomes from multicenter randomized controlled trial (KLASS-02). J Clin Oncol 2016;34:asbtr 4062.

5. Okabe H, Tsunoda S, Obama K, Tanaka E, Hisamori S, et al. Feasibility of laparoscopic radical gastrectomy for gastric cancer of clinical stage II or higher: early outcomes in a phase II study (KUGC04). Ann Surg Oncol 2016;23:S516-23.

6. Li Q, Wang J, Zhang G, Wang J, Yang B, et al. Feasibility and safety comparison of laparoscopy-assisted versus open gastrectomy for advanced gastric carcinoma with D2 lymphadenectomy. Jpn J Clin Oncol 2016;46:323-8.

7. Hao Y, Yu P, Qian F, Zhao Y, Shi Y, et al. Comparison of laparoscopy-assisted and open radical gastrectomy for advanced gastric cancer: a retrospective study in a single minimally invasive surgery center. Medicine 2016;95:25.

8. Lin JX, Huang CM, Zheng CH, Li P, Xie JW, et al. Is all advanced gastric cancer suitable for laparoscopy-assisted gastrectomy with extended lymphadenectomy? A case-control study using a propensity score method. Ann Surg Oncol 2016;23:1252-60.

9. Lee S, Lee H, Lee J. Feasibility and safety of totally laparoscopic radical gastrectomy for advanced gastric cancer: comparison with early gastric cancer. J Gastric Cancer 2018;18:152-60.

10. Li Z, Li B, Bai B, Yu P, Lian B, et al. Long-term outcomes of laparoscopic versus open D2 gastrectomy for advanced gastric cancer. Surgical Oncology 2018;27:441-8.

11. Park YK, Yoon HM, Kim YW, Park JY, Ryu KW, et al. Laparoscopy-assisted versus open D2 distal gastrectomy for advanced gastric cancer: results from a randomized phase II multicenter clinical trial (COACT 1001). Ann Surg 2018;267:638-45.

12. Kinoshita T, Uyama I, Terashima M, Noshiro H, Nagai E, et al. Long-term outcomes of laparoscopic versus open surgery for clinical stage II/III gastric cancer: a multicenter cohort study in Japan (LOC-A study). Ann Surg 2018; doi: 10.1097/SLA.0000000000002768.

13. Xu Y, Hua J, Li J, Shi L, Xue H, et al. Long-term outcomes of laparoscopic versus open gastrectomy for advanced gastric cancer: a large cohort study. Am J Surg 2019;217:750-6.

14. Kurokawa Y, Doki Y, Mizusawa J, Terashima M, Katai H, et al. Bursectomy versus omentectomy alone for resectable gastric cancer (JCOG1001): a phase 3, open-label, randomised controlled trial. Lancet Gastroenterol Hepatol 2018;3:460-8.

15. Etoh T, Sasako M, Ishikawa K, Katai H, Sano T, et al. Extranodal metastasis is an indicator of poor prognosis in patients with gastric carcinoma. Br J Surg 2006;93:369-73.

16. Lee IS, Park YS, Ryu MH, Song MJ, Yook JH, et al. Impact of extranodal extension on prognosis in lymph node-positive gastric cancer. Br J Surg 2014;101:1576-84.

17. Tsuburaya A, Mizusawa J, Tanaka Y, Fukushima N, Nashimoto A, et al. Neoadjuvant chemotherapy with S-1 and cisplatin followed by D2 gastrectomy with para-aortic lymph node dissection for gastric cancer with extensive lymph node metastasis. Br J Surg 2014;101:653-60.

18. Iwasaki Y, Terashima M, Mizusawa J, Katayama H, Nakamura K, et al. Randomized phase III trial of gastrectomy with or without neoadjuvant S-1 plus cisplatin for type 4 or large type 3 gastric cancer: Japan Clinical Oncology Group study (JCOG0501). J Clin Oncol 2018;36:abstr 4046.

19. Sano T, Sasako M, Mizusawa J, Yamamoto S, Katai H, et al. Randomized controlled trial to evaluate splenectomy in total gastrectomy for proximal gastric carcinoma. Ann Surg 2017;265:277-83.

20. Kinoshita T, Shibasaki H, Enomoto N, Sahara Y, Sunagawa H, et al. Laparoscopic splenic hilar lymph node dissection for proximal gastric cancer using integrated three-dimensional anatomic simulation software. Surg Endosc 2016;30:2613-9.

21. Uyama I, Suda K, Sato S. Textbook for Endoscopic Surgery of Fujita Health University. Nankodo Co., Ltd.; 2015. p. 64. (in Japanese)

22. Kim SY, Nam SH, Min JS, Kim MC. Laparoscopic reinforcement suture on staple-line of duodenal stump using barbed suture during laparoscopic gastrectomy for gastric cancer. Ann Surg Treat Res 2017;93:305-9.

23. Yamaguchi T, Fukunaga T, Hiki N. Laparoscopic gastric surgical procedures: distinctive (innovative) operative style of cancer institute hospital, medical view. Japan; 2011. pp. 56-69. (in Japanese)

24. Eto T, Kitano S. Laparoscope assisted distal gastrectomy. Gastroenterological Surgery 2011;34:752-60. (in Japanese)

25. Nobuoka T, Ito T, Kyuno T, Nishidate T, Imamura M, et al. Laparoscopic distal gastrectomy. Gastroenterological Surgery 2014;37:177583. (in Japanese)

26. Shimada M, Amaya S, Tatemichi K, Tawara H, Furutani Y, et al. Delicate pancreatic rotating technique using "Thoraco Cotton" in laparoscopic gastrectomy. Int J Gastroenterol Disord Ther 2017;4:133.

27. Hur H, Jeon HM, Kim W. Laparoscopic pancreas- and spleen-preserving D2 lymph node dissection in advanced (cT2) upper-third gastric cancer. Surg Oncol 2008;97:169-72.

28. Etoh T, Ohtsubo D, Misawa K, Kinoshita T, Cho H, et al. Is laparoscopic distal gastrectomy for advanced gastric cancer feasible? Shortterm outcomes from a multi-institutional, phase III study (JLSSG0901). Proceeding of the 30th annual meeting of the Japan Society for Endoscopic Surgery, 2017 Dec 7-9. Kyoto, Japan; 2017.

29. Kawaguchi Y, Shiraishi K, Akaike H, Ichikawa D. Current status of laparoscopic total gastrectomy. Ann Gastroenterol Surg 2019;3:1423. 
30. Cunningham D, Allum WH, Stennning SP, Thompson JN, Van de Velde CJ, et al. Perioperative chemotherapy versus surgery alone for resectable gastroesophageal cancer. N Engl J Med 2006;355:11-20.

31. Ychou M, Boige V, Pignon JP, Conroy T, Bouché O, et al. Perioperative chemotherapy compared with surgery alone for resectable gastroesophageal adenocarcinoma: an FNCLCC and FFCD multicenter phase III trial. J Clinic Oncol 2011;29:1715-21.

32. Li Z, Shan F, Ying X, Zhang L, Ren H, et al. Laparoscopic or open distal gastrectomy after neoadjuvant chemotherapy for advanced gastric cancer: study protocol for a randomised phase III trial. BMJ Open 2018;8:e21633.

33. Yoshida K, Yamaguchi K, Okumura N, Tanahashi T, Kodera Y. Is conversion therapy possible in stage IV gastric cancer: the proposal of new biological categories of classification. Gastric Cancer 2016;19:329-38

34. Yamaguchi K, Yoshida K, Tanahashi T, Takahashi T, Matsuhashi N, et al. The long-term survival of stage IV gastric cancer patients with conversion therapy. Gastric Cancer 2018;21:315-23.

35. Terashima M, Tokunaga M, Tanizawa Y, Bando E, Kawamura T, et al. Robotic surgery for gastric cancer. Gastric Cancer 2015;18:449-57.

36. Kinoshita T, Kaito A. Current status and future perspectives of laparoscopic radical surgery for advanced gastric cancer. Transl Gastroenterol Hepatol 2017;2:43.

37. Kim MC, Heo GU, Jung GJ. Robotic gastrectomy for gastric cancer: surgical techniques and clinical merits. Surg Endosc 2010;24:610-5.

38. Hashizume M, Sugimachi K. Robot-assisted gastric surgery. Surg Clin North Am 2003;83:1429-44.

39. Song J, Oh SJ, Kang WH, Hyung WJ, Choi SH, et al. Robot-assisted gastrectomy with lymph node dissection for gastric cancer: lessons learned from an initial 100 consecutive procedures. Ann Surg 2009;249:927-32.

40. Li Z, Li J, Li B, Bai B, Liu Y, et al. Robotic versus laparoscopic gastrictomy with D2 lymph node dissection for advanced gastric cancer: a propensity score-matched analysis. Cancer Manag Res 2018;10:705-14.

41. Kim HI, Han SU, Yang HK, Kim YW, Lee HJ, et al. Multicenter prospective comparative study of robotic versus laparoscopic gastrectomyfor gastric adenocarcinoma. Ann Surg 2016;263:103-9. 